\title{
Review of Energetic Particle Generation and Electromagnetic Radiation from Intense Laser-Plasma Interactions at the Institute of Physics, Chinese Academy of Sciences*)
}

\author{
Yutong $\mathrm{LI}^{1)}$, Zhengming SHENG ${ }^{1,2)}$, Liming $\mathrm{CHEN}^{1)}$, Xin $\mathrm{LU}^{1)}$, Jinglong MA ${ }^{1)}$, \\ Zhaohua WANG ${ }^{1)}$, Quanli DONG ${ }^{1)}$, Zhiyi WEI ${ }^{1)}$ and Jie ZHANG ${ }^{1,2)}$ \\ ${ }^{1)}$ Beijing National Laboratory for Condensed Matter Physics, Institute of Physics, Chinese Academy of Sciences, \\ Beijing 100190, China \\ ${ }^{2)}$ Department of Physics, Shanghai Jiao Tong University, Shanghai 200240, China
}

(Received 31 January 2009 / Accepted 23 March 2009)

\begin{abstract}
This review covers recent developments in multi-hundred-TW femtosecond laser systems and progress in intense laser-plasma interactions at the Institute of Physics, Chinese Academy of Sciences. The peak power of the institute's Xtreme Light III (XL-III) laser system has been upgraded from 350 to $725 \mathrm{TW}$, and the system used to observe the lateral transport of fast electrons due to magnetic and electrostatic fields at target surfaces. The surface fields cause fast-electron beams to be emitted along the front and rear target surfaces. Confinement and guiding of fast-electron propagation is demonstrated with wire- or wedge-shaped targets. Longitudinal transport is detected by optical and ion emission. Numerical simulations show that quasi-monoenergetic ion beams can be generated by collisionless electrostatic shock acceleration and phase-stable acceleration with a circularly polarized laser field. A new mechanism for high-power $\mathrm{THz}$ emission with plasmas as media is proposed; it can be used to obtain a single-cycle $\mathrm{THz}$ emission. The conversion efficiency from laser energy to $\mathrm{K}_{\alpha} \mathrm{X}$-ray emission in a laser-copper target interaction is increased to $10^{-4}$ using high-contrast laser pulses.
\end{abstract}

(c) 2009 The Japan Society of Plasma Science and Nuclear Fusion Research

Keywords: ultraintense laser-plasma interaction, femtosecond laser pulse, fast electron, energetic ion, $\mathrm{THz}$ radiation, ultrafast $\mathrm{x}$-ray emission

DOI: $10.1585 /$ pfr.4.023

\section{Introduction}

Since the advent of the chirped-pulse amplification (CPA) technique, there has been rapid progress in the development of high-power, ultrashort laser systems. To date, Ti:sapphire CPA laser systems with a peak power of hundreds of terawatts and a pulse duration of tens of femtoseconds are available in many laboratories worldwide. Research frontiers in high-field, laser-matter interactions are being explored, resulting in great opportunities and numerous potential applications related to, for example, particle acceleration, novel radiation sources, laser fusion, and even fundamental physics.

In this paper, we review our recent progress in building intense laser systems and in studying electron and ion acceleration, and short-pulse $\mathrm{THz}$ and $\mathrm{X}$-ray emission, in the interactions of intense laser pulses with plasmas.

\section{Upgrading of the XL-III Laser Sys- tem}

The 350-TW short-pulse laser system, Xtreme Light (XL)-III, was upgraded recently. Its peak power with only

author's e-mail: jzhang@aphy.iphy.ac.cn

*) This article is based on the invited talk at the 14th International Congress on Plasma Physics (ICPP2008). three amplifier stages now reaches $725 \mathrm{TW}$, realized by optimizing its efficiency. The system consists of a homemade Ti:sapphire oscillator, an Öffner stretcher, a regenerative amplifier, two multipass amplifiers, and a vacuum compressor. Seed pulses are derived from a Ti:sapphire Kerr-lensing mode-locked oscillator pumped by a $5-\mathrm{W}$ frequency-doubled continuous-wave $(\mathrm{CW})$ diode-pumped $\mathrm{Nd}: \mathrm{YVO}_{4}$ laser (Spectra-Physics, Inc.). The oscillator produces a 6-nJ train of pulses (duration $20 \mathrm{fs}$, rep. rate $82 \mathrm{MHz})$.

After passing through a Faraday isolator, the pulse train is stretched by a typical all-reflective Öffner-triplet stretcher. Our stretcher consists of a single 1480grooves/mm gold-coated grating, a concave spherical mirror, and a convex spherical mirror.

The stretched pulses are sent into the first-stage amplifier. This regenerative amplifier is pumped by a 50 $\mathrm{mJ} / 532-\mathrm{nm}$ laser at $10 \mathrm{~Hz}$, and provides a net gain of approximately $10^{7}$; about $4 \mathrm{~mJ}$ of energy is obtained, in 600-ps pulses measured full width at half maximum (FWHM). Because of the gain-narrowing effect in the preamplifier, we use a commercial acousto-optic modulator (DAZZLER ${ }^{\mathrm{TM}}$ WB-800, Fastlite, Inc.) to shape the spectrum, thus broadening the bandwidth to $36 \mathrm{~nm}$. To 
minimize the prepulse, we use two crossed Glan-laser prisms and a Pockels cell as a single-pulse selector after the regenerative amplifier.

The beam is expanded to $12 \mathrm{~mm}$ and sent into the second-stage amplifier. In this six-pass amplifier, a Ti:sapphire crystal $(25 \times 15 \mathrm{~mm})$ is pumped from both sides by a $2.2-\mathrm{J} / 532-\mathrm{nm}$ laser, and about $340 \mathrm{~mJ}$ of energy is obtained.

The beam size is further expanded to $60 \mathrm{~mm}$ with a telescope set in a vacuum chamber and used as an image relay, then injected into the third-stage amplifier. In this five-pass amplifier, a Ti:sapphire crystal disk $(85 \times 20 \mathrm{~mm})$ with antireflection coating on both surfaces is pumped by a 100-J laser, and about $43.9 \mathrm{~J}$ of energy is obtained. To eliminate parasitic oscillation lasing and amplified spontaneous emission, the crystal is held with an absorptive polymer thermoplastic (Cargille Laboratories, Inc.) cladding.

After amplification, the output beam is up-collimated with a telescope and sent into the compressor, where the beam size is further expanded to $120 \mathrm{~mm}$. The compressor consists of four gold-coated holographic gratings (JobinYvon, Inc.) with a groove of 1480 lines $/ \mathrm{mm}$. The total transmission of the telescope and compressor system is about $52.3 \%$, producing a compressed output pulse energy of $22.5 \mathrm{~J}$ with a pulse duration of $31 \mathrm{fs}$, reaching a peak power of $725 \mathrm{TW}$.

\section{Fast-Electron Generation and Transport}

Studies on laser-produced fast electrons have attracted great interest worldwide because of their prospective applications in fast ignition in inertial confinement fusion [1], high-energy ion generation [2], x-ray emission [3], and others. Figure 1 shows a schematic view of fast-electron generation and transport. When a high-intensity laser pulse is focused onto a solid target with a focal spot a few micrometers in diameter, a large number of electrons can be accelerated to very high energies, forming so-called fast or hot electrons. For example, fast electrons can be generated by $J \times B$ heating [4] and vacuum heating [5] in a plasma with steep electron-density gradients, while, by stochastic heating [6] and various parametric instabilities [7] in a plasma with large scale length of electron-density.

After the fast electrons gain sufficient energy, some of them are ejected backward into vacuum from the plasma in front of the target. The rest are transported into the overdense plasma and cold target region, where some of them may escape from the rear target surface. Transport can be longitudinal (in the direction of laser propagation) and/or lateral (along the target surfaces) [8-10].

In our recent laser-solid experiments with the 20-TW XL-II laser system, we find collimated electron beams emitted along the front surface at a large laser incidence angle $\left(>60^{\circ}\right)[11]$. This agrees with the particle-in-cell (PIC) simulations performed by Nakamura et al. [12] and

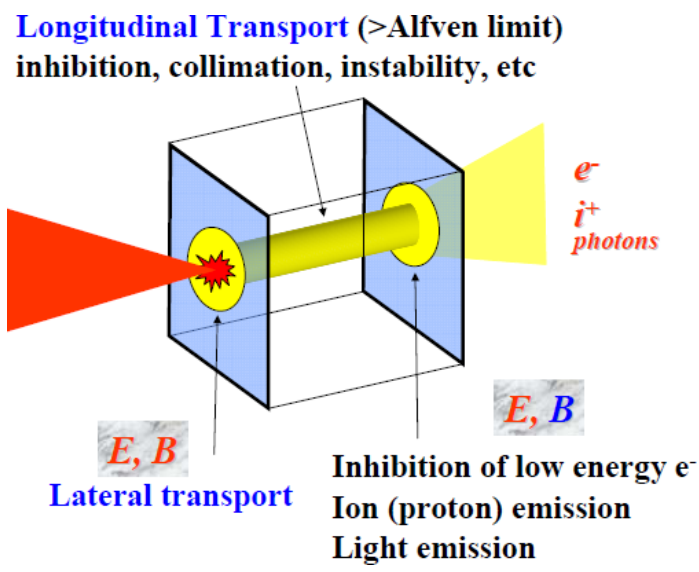

Fig. 1 Schematic view of fast-electron generation and transport. The spontaneous electrostatic fields $E$ and magnetic fields $B$ at the target surface and in the target bulk are important for electron transport. The fields at the front surface can induce lateral transport. The fields in the bulk can strongly inhibit longitudinal electron transport. The fields at the rear surface can accelerate ions and restrain low-energy electrons from escaping into the vacuum.

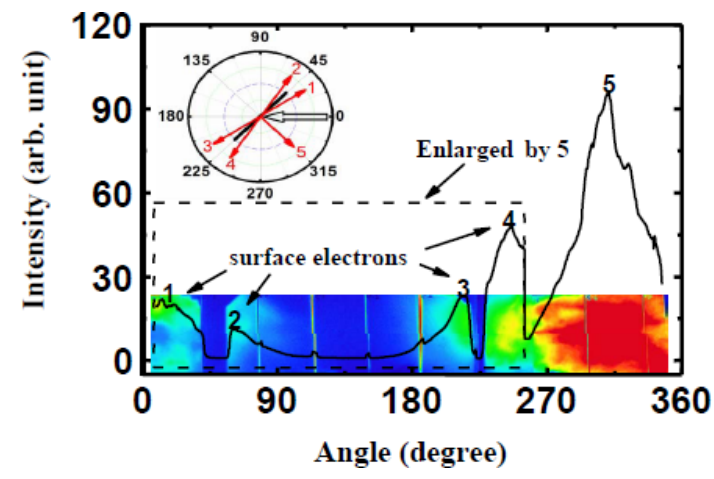

Fig. 2 Angular distribution of fast electrons $(E>900 \mathrm{keV})$ for a plastic target (thickness $30 \mu \mathrm{m}$ ) at a laser intensity of $1.5 \times 10^{18} \mathrm{~W} / \mathrm{cm}^{2}$. For clarity, the angular distributions enclosed by the dashed box are enlarged by a factor of 5. The inset shows the laser incidence direction (hollow arrow) and each group of fast-electron beams (red numbered arrows). Four electron beams (1-4) are emitted along the target surface and the other beam (5) is emitted in the target normal direction.

Sentoku et al. [13], and indicates that electron beams form because of confinement of the surface quasistatic electromagnetic fields. This observation experimentally demonstrates the feasibility of electron guiding in the coneguided fast-ignition scheme proposed by Kodama [14].

When laser incidence angles are moderate, four groups of collimated fast-electron beams along the front and rear target surfaces are observed, as shown in Fig. $2[15,16]$. This multipeak characterization is independent of laser polarization and is observed for both aluminum and plastic foil targets. PIC simulations suggest 


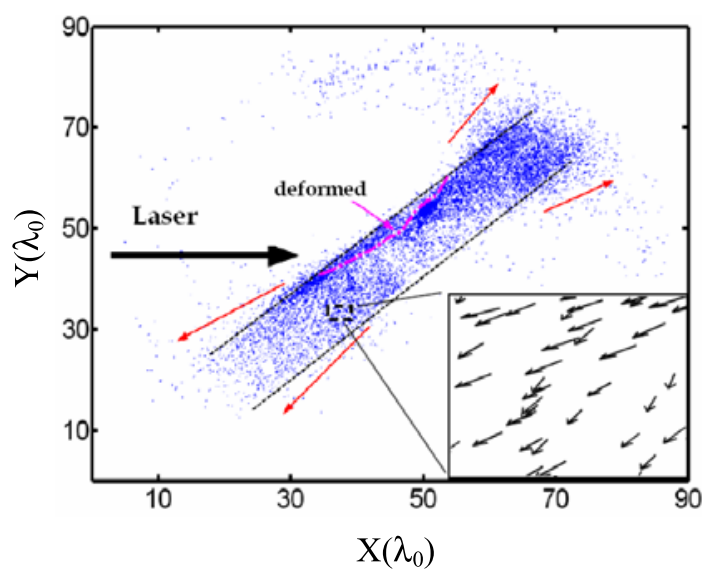

Fig. 3 Phase space distribution of fast electrons $(E>900 \mathrm{keV})$ at $100 T_{0}$, simulated by a 2D PIC code. The black solid arrow shows the laser incidence direction. The black dashed line is the original target surface. The pink dotdashed line is the deformed target surface. The thin red arrows show the fast electrons along the target surfaces. The inset shows an enlarged view of fast electrons with the movement directions.

that such fast electrons are generated when the target surface is deformed by the strong ponderomotive force of the laser pulses and then constrained by the quasistatic electromagnetic fields (see Fig. 3).

Simulations suggest that the strong electrostatic or magnetic fields induced at the target surfaces can also be used to guide fast-electron propagation [17]. Measurement of ultraviolet optical emission [18] and X-ray emission [19] from shaped targets indicates the feasibility of guiding fast electrons through the fields at the nonirradiated target surfaces. By directly observing fast-electron emission from the interaction of short laser pulses with various shaped solid targets, we also find that the angular distributions of the fast electrons are highly dependent on the target shape [20].

In our experiments, we focus the pulse from the XLII laser system using an f/3.5 off-axis parabolic mirror onto planar and wedge-shaped copper targets. The standard planar target is a copper disk (thickness $65 \mu \mathrm{m}$, diameter $1000 \mu \mathrm{m}$ ), and the wedge-shaped target has the following dimensions: tip angle $25^{\circ}$, height $150 \mu \mathrm{m}$. The angular distributions of the forward fast electrons behind the targets are measured by an array of imaging plate (IP) stacks. Figure 4 shows the measured angular distributions for the planar and wedge-shaped targets. Fast electrons $(E>600 \mathrm{keV})$ are emitted with a single smooth peak near the target normal for the planar target, and with double peaks on both sides of the isosceles surfaces for the wedgeshaped target. However, a large number of $E>120 \mathrm{keV}$ electrons are emitted in the tip direction, resulting in a distribution without obvious concavity at the central part. This is much different than for the $E>600 \mathrm{keV}$ electrons. (a)

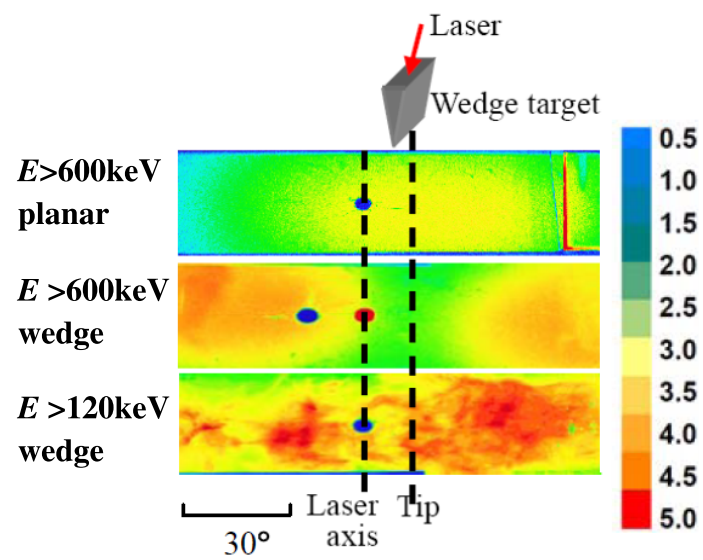

Fig. 4 Angular distributions of fast electrons from (a) planar target, $E>600 \mathrm{keV}$; (b) wedge-shaped target, $E>600 \mathrm{keV}$; (c) wedge-shaped target, $E>120 \mathrm{keV}$. The top surface opposite the wedge-target tip is irradiated by the laser pulse.

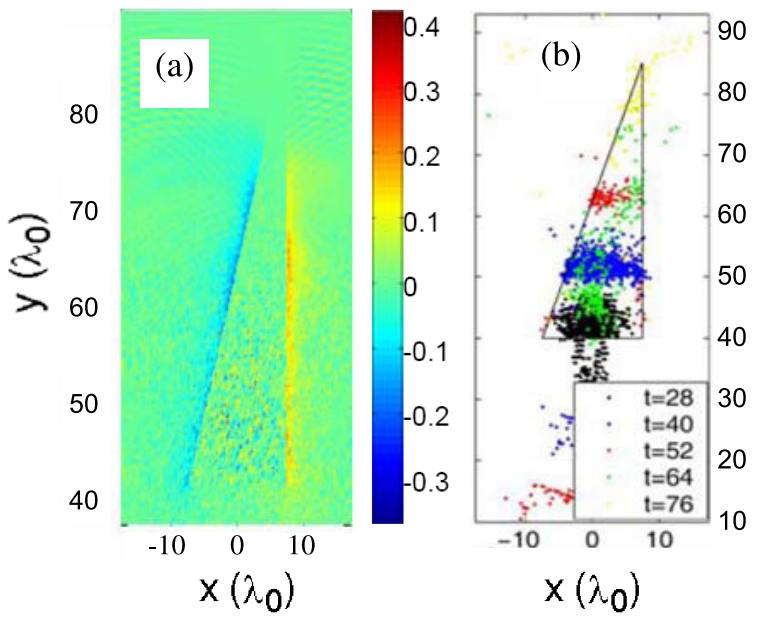

Fig. 5 Snapshots of the electrostatic field $E_{x}$ (normalized by the incident laser amplitude) obtained by 2D PIC simulations at $t=60$ laser cycles (a). Distributions of fast electrons with relativistic factor $\gamma>4$ at $t=28,40,52,64$, and 76 laser cycles (b). Laser is incident from the bottom.

Fast-electron transport in a right-angle wedge-shaped plasma slab (tip angle $18.4^{\circ}$ ) was simulated with a 2D PIC code, in which binary collisions were considered. Figure 5 shows the spatial distributions of the electrostatic fields in the $X$ direction $\left(E_{x}\right)$ at 60 laser cycles and the distributions of the propagating fast electrons at different times. The fields are normalized by the incident laser amplitude $E_{0}$. One can see that strong electrostatic fields are set up at the two side surfaces of the wedge. These fields effectively inhibit the electrons from escaping into vacuum, resulting in electron guiding to the tip.

A large fast-electron current in the longitudinal direction induces electrostatic and magnetic fields inside the target bulk. The bulk fields, in turn, affect fast-electron trans- 


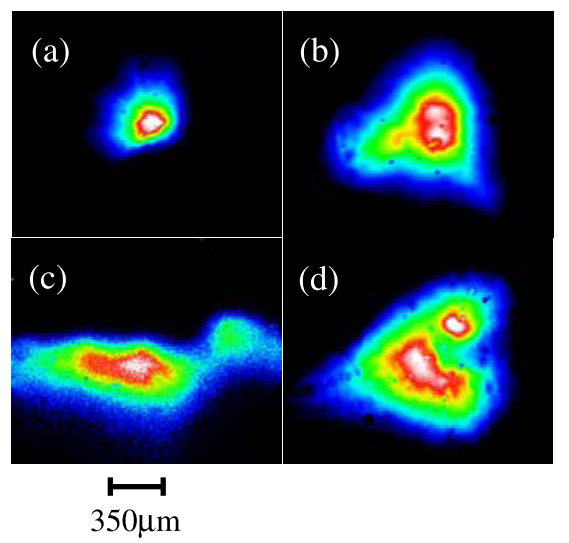

Fig. 6 TR images for targets with different transport layers: (a) $100 \mu \mathrm{m} \mathrm{Ta}$; (b) $20 \mu \mathrm{m} \mathrm{Ta}+60 \mu \mathrm{m} \mathrm{CH}+20 \mu \mathrm{m} \mathrm{Al}$; (c) $10 \mu \mathrm{m} \mathrm{Ta}+500 \mu \mathrm{m}$ foam $\left(160 \mathrm{mg} / \mathrm{cm}^{3}\right)+5 \mu \mathrm{m} \mathrm{Al}$; (d) $20 \mu \mathrm{m} \mathrm{Ta}+75 \mu \mathrm{m}$ vacuum gap $+20 \mu \mathrm{m} \mathrm{Al}$.

Table 1 Laser intensity, peak count, and integrated count for the four types of targets shown in Fig. 6 .

\begin{tabular}{lllll}
\hline & (a) & (b) & (c) & (d) \\
\hline $\begin{array}{l}\text { Laser intensity } \\
\left(10^{18} \mathrm{~W} / \mathrm{cm}^{2}\right)\end{array}$ & 2.44 & 2.67 & 1.34 & 2.12 \\
$\begin{array}{l}\text { Peak count } \\
\text { (arb. units) }\end{array}$ & $1.2 \times$ & $4.3 \times$ & $1.7 \times$ & $9.7 \times$ \\
$\begin{array}{l}\text { Integrated count } \\
\text { (arb. units) }\end{array}$ & 75880 & 18597 & 331 & 3841 \\
\hline
\end{tabular}

port. Feedback processes between the fast electrons and the bulk fields can lead to self-organized propagation of the fast-electron current. Effective energy loss [21,22] and beam breakup [23,24] can occur.

When the fast electrons reach the target rear surface, the induced strong electric fields can accelerate ions (preferably protons) by the target normal sheath acceleration (TNSA) mechanism [2]. When the fast electrons pass the rear target surface-vacuum boundary, transition radiation (TR) occurs [25]. Therefore, the characteristics of the proton beam and the optical light emission at the rear surface correlate with fast-electron transport.

Recently, we measured the TR light and proton emission from the back surface of sandwich-like targets to deduce information about the electron transport. Figure 6 shows the optical emission images obtained using a Ta solid target and various sandwiched targets with plastic, foam, and vacuum gaps as transport layers. Note that the images are shown with different intensity scales to display the beam structures clearly. Peak and integrated counts for the TR signals are shown in Table 1. For the Ta solid target (thickness $100 \mu \mathrm{m}$ ), the beam is homogenous. For the
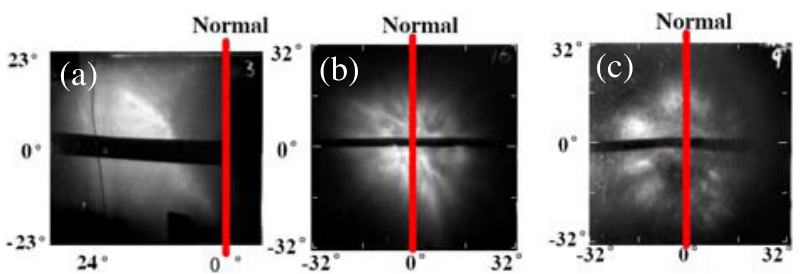

Fig. 7 Angular distributions of the proton beam measured behind the following targets: (a) $20 \mu \mathrm{m} \mathrm{Ta}$; (b) $20 \mu \mathrm{m} \mathrm{Ta}+$ $60 \mu \mathrm{m} \mathrm{CH}+20 \mu \mathrm{m} \mathrm{Al}$; (c) $20 \mu \mathrm{m} \mathrm{Ta}+75 \mu \mathrm{m}$ vacuum gap $+20 \mu \mathrm{m} \mathrm{Al}$.

sandwiched target with plastic (thickness $60 \mu \mathrm{m}$ ), the beam is larger and inhomogeneous, and the TR light signals are accordingly weaker. For the sandwiched target with foam (thickness $500 \mu \mathrm{m}$, density $160 \mathrm{mg} / \mathrm{cm}^{3}$ ), the signal intensity is further reduced by about two orders of magnitude, and the beam is much distorted and broken up. For the sandwiched target with vacuum gap, the beam is also broken up.

Figure 7 shows the angular distributions of the proton beam behind the targets measured by CR-39 detector. The mean proton numbers for the single $20-\mu \mathrm{m}$ Ta, $60-\mu \mathrm{m} \mathrm{CH}$, and $75-\mu \mathrm{m}$ vacuum gap transport targets are approximately $8 \times 10^{7}, 4 \times 10^{7}$, and $5 \times 10^{6} / \mathrm{sr}$, respectively. The proton beam behind the single Ta foil does not present many fine structures, while the proton beams for the targets with the $\mathrm{CH}$ layer and the vacuum gap are very inhomogeneous with ripples and spots.

From Figs. 6 and 7, one can see that the intensities of the TR signals and the proton beams are decreased and the patterns are broken up for the sandwiched targets with dielectric, low foam, and vacuum transport layers. This indicates that effective inhibition and breakup occur for the fast-electron beam during transport in the targets.

\section{Proton and Ion Acceleration}

In recent years, with the rapid development of laser technology, ion acceleration has received extensive attention in the area of laser plasma interactions [2]. We observe ring-like angular distributions of proton beams when a femtosecond laser pulse with a large prepulse is used [26]. Bulk acceleration of ions with low-density foams as targets has also been demonstrated [27].

For laser-solid interactions, when the laser intensity is relatively low $\left(<10^{19} \mathrm{~W} / \mathrm{cm}^{2}\right)$, ions are dominantly accelerated by the sheath field generated near the target surfaces because of charge separation. When the intensity is high (> $10^{22} \mathrm{~W} / \mathrm{cm}^{2}$ ), so-called laser-piston acceleration occurs [28], where the radiation pressure is dominant and the laser energy is transferred efficiently into fast ions. When the laser intensity is in the moderate range, another type of ion acceleration may appear, in which a collisionless electrostatic shock (CES) is generated in the target and the ions 
in front of it can be reflected and get accelerated [29, 30]. Shock acceleration deserves to receive much more attention since the energy spectrum of the ions accelerated by this mechanism is ideally monoenergetic, which is useful for all types of applications. It is also worth noting that shock acceleration can be achieved with the laser parameters currently available. Upon revisiting this problem to find the laser and target parameter regime suitable for experiments [31-34], we find that shock generation depends significantly upon target parameters such as density, ion mass, and thickness. It also depends upon laser intensity and related preplasma conditions at both the front and rear sides of the target. Simulation results suggest that CESs can be generated over a wide range of target and laser parameters, even for a laser-pulse intensity of $10^{18} \mathrm{~W} / \mathrm{cm}^{2}$, as long as the parameters are carefully selected. This makes it possible to demonstrate shock acceleration at low laser intensities. In experiments to distinguish shock acceleration from sheath-field acceleration, we propose to use a sandwich target. For this purpose, we investigate shock propagation in such targets. Our simulation shows that the shock is destroyed if the difference in charge-to-mass ratio $R$ of ions in the two neighboring targets is large.

As evident from intense laser interactions with solid targets, ions are always accelerated by the sheath field at both the front and rear sides of a target. Although some ions in the target can be accelerated by the CES wave, they also experience sheath-field acceleration as they move to the rear surface of the target. Therefore, it is difficult to distinguish shock acceleration from sheath-field acceleration experimentally. We therefore suggest that using a sandwich target (Fig. 8 (a)), one can unequivocally demonstrate shock acceleration by detecting the unique ions in the middle layer. Since there is no sheath field established around the middle layer, the unique ions in the central layer cannot move to the rear side of a sandwich target unless they are accelerated by a shock wave. Of course, the thickness of the outer layers must be carefully selected to ensure that the sheath field does not expand to the middle layer. We find that the charge-to-mass ratio $R$ for ions near the interface between the front and middle layers deserves special attention. Figures 8 (b) and 8 (c) show a shock propagating through the interface; however, in certain conditions, the shock may evolve into a solitary wave that cannot accelerate the ions in the second layer. Therefore, to demonstrate shock acceleration experimentally, one cannot use sandwich targets with a large difference in $R$. We suggest using a doped target in which the middle layer is adulterated into many light ions. Our simulations show that when ions with high $R$ are adulterated into the center region of a target that is composed mostly of ions with low $R$, the ions with high $R$ can be accelerated by the shocks and can even bounce between shocks if one uses two laser pulses to inject the target from both the front and back of the target. The final velocity of the light ions (high $R$ ) is related to the bounce times and is determined only by the shock potential and
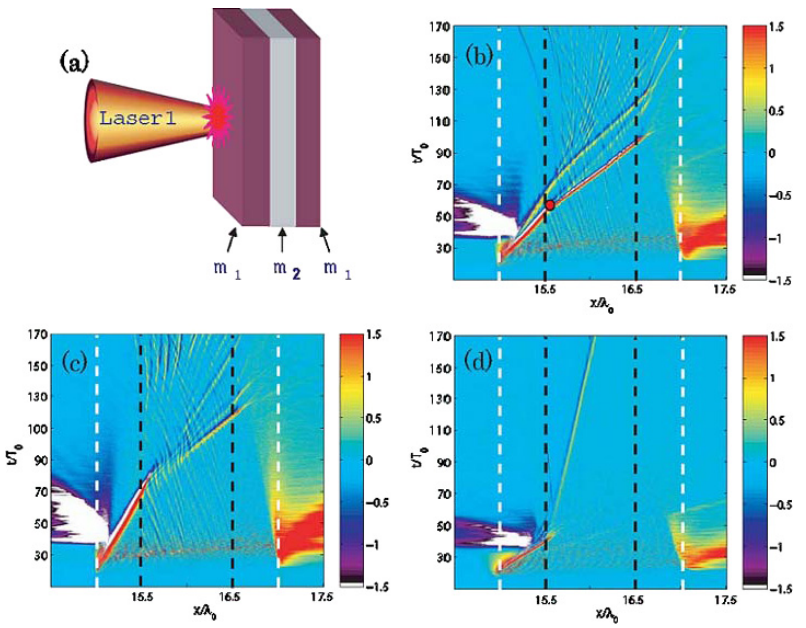

Fig. 8 (a) Schematic of the experimental setup proposed to demonstrate the mechanism for shock acceleration. (b)(d) Shock propagating through a two-target interface. The figures show the spatial and temporal evolution of the longitudinal electric fields (normalized by incident laser amplitude $E_{0}$ ) of the shocks. Masses of the ions in the first $\left(m_{1}\right)$ and second layer $\left(m_{2}\right)$ are as follows: (b) $m_{1}=7344, m_{2}=1836$; (c) $m_{1}=18360, m_{2}=1836$; (d) $m_{1}=1836, m_{2}=18360$. The white dashed lines are the left and right boundaries of the target; the black dashed lines are the interfaces of the neighboring layers.

not its speed.

For collisionless shock-wave acceleration of protons, although their energy spectrum is quasi-monoenergetic, their maximum energy is limited to about $2 m_{\mathrm{i}} v_{\mathrm{s}}^{2}$, where $v_{\mathrm{s}}$ is the shock speed. A new scheme called phase-stable acceleration of protons is proposed [34]. One of the key points of this proposal is the need to use circularly polarized laser pulses. When the initial target density $n_{0}$ and thickness $d$ satisfy $\left(n_{0} / n_{\mathrm{c}}\right)\left(d / \lambda_{\mathrm{L}}\right) \sim a_{\mathrm{L}}$, where $a_{\mathrm{L}}, \lambda_{\mathrm{L}}$, and $n_{\mathrm{c}}$ are the normalized laser amplitude, the laser wavelength in vacuum, and the critical density of the incident laser pulse, respectively, a quasi-equilibrium for electrons is established as a result of the light pressure and electrostatic field built up at the interacting front of the laser pulse. The ions located within the skin depth of the laser pulse can be synchronously accelerated and bunched in the push-andpull processes by the electrostatic field, and thus a monoenergetic, high-intensity proton beam can be generated. Protons can be accelerated to $>300 \mathrm{MeV}$ with an energy spread of only a few percent by a laser pulse with a focused intensity of $6.8 \times 10^{19} \mathrm{~W} / \mathrm{cm}^{2}$.

Figure 9 shows snapshots of the phase space of protons in the compressed region. It shows that protons execute period oscillations. After about 40 laser cycles of interaction, the protons have accelerated to a longitudinal momentum of about $p_{x}=0.4 M c$, where $M$ is the rest mass of a proton. The reflection coefficient is about $\eta=0.38$, which implies that more than $60 \%$ of the laser 

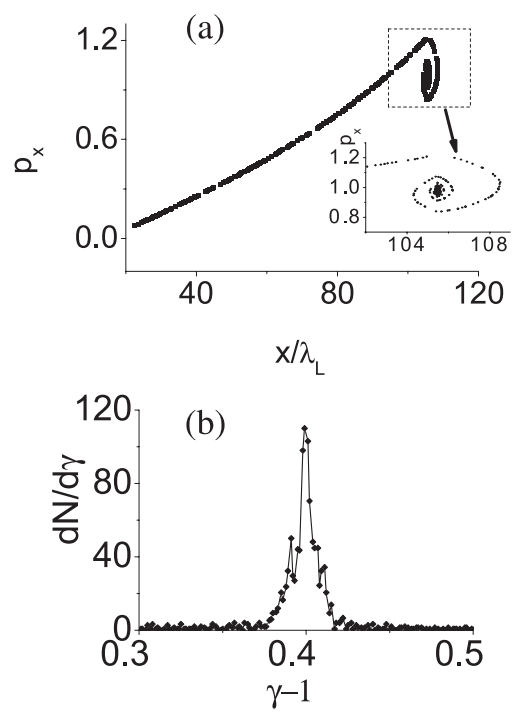

Fig. 9 (a) Phase space distribution of protons; (b) energy spectrum of protons. The results are for $t=200 T_{\mathrm{L}}$, when the laser pulse interaction is almost terminated. The plasma slab has a steep density profile with initial density $n_{0} / n_{\mathrm{c}}=10$ and thickness $L=0.2 \lambda_{\mathrm{L}}$. The laser pulse has a normalized laser peak amplitude $a_{\mathrm{L}}=5$ and duration $100 T_{\mathrm{L}}$.

energy is converted into electron and proton energy. Of the absorbed laser energy, $>10 \%$ is contained in the accelerated protons. This result is obtained from 1D PIC simulation. However, for multidimensional geometry, acceleration efficiency will be significantly reduced and the energy spectrum will also be broadened. To overcome this multidimensional effect, one can use supergaussian laser pulses with a large spot size or even combine laser pulses with super-Hermite and Gaussian modes to improve the ion acceleration and transverse collimation.

\section{THz Radiation}

Intense laser-plasma interactions can produce various novel radiation sources from terahertz radiation $(1 \mathrm{THz}=$ $10^{12} \mathrm{~Hz}$ ) to gamma rays $[35,36]$. Currently, terahertz radiation from laser-irradiated targets attracts much interest. $\mathrm{THz}$ science and technology have become a frontier of research for disciplines crossing the fields of physics, chemistry, biology, materials science, and medicine. Based on the optical schemes, $\mathrm{THz}$ emission can be generated by optical rectification, optical conductive antenna, nonlinear difference frequency mixing, tunable parametric oscillators, optical Cherenkov radiation, etc. However, because of the damage limit of optical materials, one cannot irradiate these optical materials with very-high-power lasers. With these schemes, it is difficult to obtain high-power $\mathrm{THz}$ emission at the megawatt level, as is needed for many applications and for the study of high-field and nonlinear physics in the $\mathrm{THz}$ regime in semiconductors, insulators, superconductors, etc. [37]. Currently, MW narrow-band
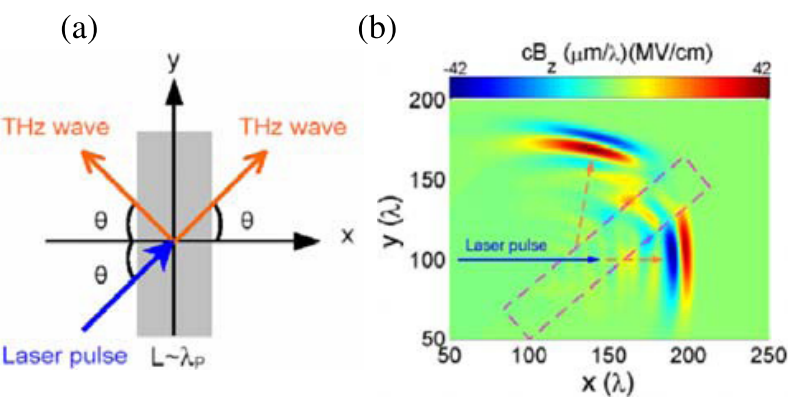

Fig. 10 (a) Schematic diagram of $\mathrm{THz}$ emission from a plasma oscillator excited by a laser pulse obliquely incident on a few-wavelength-thick uniform plasma slab. $\mathrm{THz}$ waves are radiated in both specular reflection and transmission directions. (b) 2D spatial plot of the pure $\mathrm{THz}$ magnetic field $B_{z}$ in 2D PIC simulations. The dashed arrows show the $\mathrm{THz}$ emission directions, one along the laser propagation and the other along the specular reflection direction.

THz sources are obtained from free-electron lasers. Broadband $\mathrm{THz}$ sources from ultrashort electron bunches, either from linac or laser wakefields, are also suggested [38, 39].

On the other hand, plasma as a nonlinear optical medium does not have a damage limit by high-power lasers. Thus, by irradiating plasma with such laser pulses, one can, in principle, obtain very-high-power $\mathrm{THz}$ pulses. In fact, in the 1990s, Hamster et al. observed THz emission by irradiating ultrashort laser pulses of $100 \mathrm{fs}$ onto solid and gas targets [40]. Later, $\mathrm{THz}$ emission from Cherenkov wakes in magnetized plasmas driven by ultrashort laser pulses was proposed [41]. Recently, we suggested a new mechanism for $\mathrm{THz}$ emission based on the excitation of laser wakefields [42-46]. This emission is produced through linear-mode conversion from a highamplitude laser wakefield driven in inhomogeneous plasmas. This scheme can provide emission with power on the order of tens of MW and field amplitudes up to a few $\mathrm{GV} / \mathrm{m}$, and still allow for both compactness and relatively high efficiency using TW ultrashort laser pulses. Furthermore, it is suggested that single-cycle THz emission can be produced with thin underdense plasma layers (a few $\mathrm{THz}$ wavelengths thick) irradiated by laser pulses [47].

In addition, ionizing gas targets are also potentially high-power $\mathrm{THz}$ sources when irradiated by laser pulses at moderate intensities such as $10^{15-16} \mathrm{~W} / \mathrm{cm}^{2}$ [48-51]. THz emission can be produced with either one or two laser pulses. For example, laser filaments are observed to emit electromagnetic radiation in the sub- $\mathrm{THz}$ region. Both transverse and very directional forward and backward emissions have been observed. There are several different explanations for these observed emissions, such as four-wave mixing [48], ionization-induced current models [49], Cherenkov emission from the ionization front moving faster than the speed of light in the medium [50], and transition-Cherenkov emission from the plasma space 
charge moving behind the ionization front [51]. Here, we study the generation of $\mathrm{THz}$ dipoles by tunneling and above-barrier ionization, and find that the photoelectrons perform space-charge oscillations and radiate $\mathrm{THz}$ waves at the local plasma frequency, depending on gas density [52]. The THz field amplitude scales linearly with the laser amplitude, which however holds only when the latter is at a moderate level. To overcome this limitation, we propose a scheme using chirped laser pulses irradiating on tenuous gas or plasma targets, which can generate $\mathrm{THz}$ pulses with amplitude 10 to 100 times larger than those from the well-known two-color laser scheme [53], enabling one to obtain a $\mathrm{THz}$ field up to $10 \mathrm{MV} / \mathrm{cm}$ with incident laser at approximately $10^{16} \mathrm{~W} / \mathrm{cm}^{2}$.

\section{Short-Pulse X-ray Emission}

In intense femtosecond laser-solid interactions, fast electrons, generated via collective absorption mechanisms such as resonant absorption (RA) [7] or vacuum heating (VH) [5], penetrate the solid target and produce hard x-ray emission via K-shell ionization and bremsstrahlung [54]. This type of intense and ultrafast hard x-ray source has a number of interesting applications for medical imaging [55]. It is found that the yield of the hard x-ray emission is not so high with increasing laser intensity for laserpulse durations of tens of femtoseconds when low-contrast laser pulses are used [56].

Recently, we showed that hard x-ray emission can be enhanced using high-contrast femtosecond laser pulses [57]. The experiment was performed using the XLII laser system. A type I potassium dideuterium phosphate (KDP) frequency-doubling crystal (thickness $1 \mathrm{~mm}$ ) was used to obtain a 400-nm second harmonic pulse. This increased the pulse contrast to $>10^{8}$ at the picosecond pedestal and $>10^{10}$ in the ns time window. The laser pulse was obliquely incident on $\mathrm{Cu}$ foil targets (thickness $5 \mu \mathrm{m}$ ) at $45^{\circ}$ by an $\mathrm{f} / 3.5$ parabola mirror (focal spot diameter $10 \mu \mathrm{m}$ FWHM). We varied the laser pulse duration from $60 \mathrm{fs}$ to $2 \mathrm{ps}$ by changing the distance between the gratings at constant laser energy. Increasing (decreasing) distance causes incomplete compensation of accumulated phase nonlinearities, resulting in negatively (positively) chirped pulses having a gentle (steep) rise time [58], as shown in Fig. 11.

Figure 12 shows the $\mathrm{Cu}$ conversion efficiency from laser energy to $\mathrm{K}_{\alpha} \mathrm{x}$-rays, $\eta_{\mathrm{K}}$, as a function of the 400 $\mathrm{nm}$ laser pulse duration with negative and positive modification. $\mathrm{Cu} \eta_{\mathrm{K}}$ with negatively modified (by $100 \mathrm{fs}$ ) pulse duration is dramatically improved; it reaches a maximum of $4 \times 10^{-4}$ and is almost 5 times greater than for the positively modified (by $100 \mathrm{fs}$ ) pulse duration. However, for s-polarized laser incidence, $\mathrm{Cu} \eta_{\mathrm{K}}$ is 3 times lower and does not show evident pulse chirp dependence. It simply decreases with increasing laser pulse duration. We find, for this regime, that $\mathrm{VH}$ is stimulated and is the main ab-

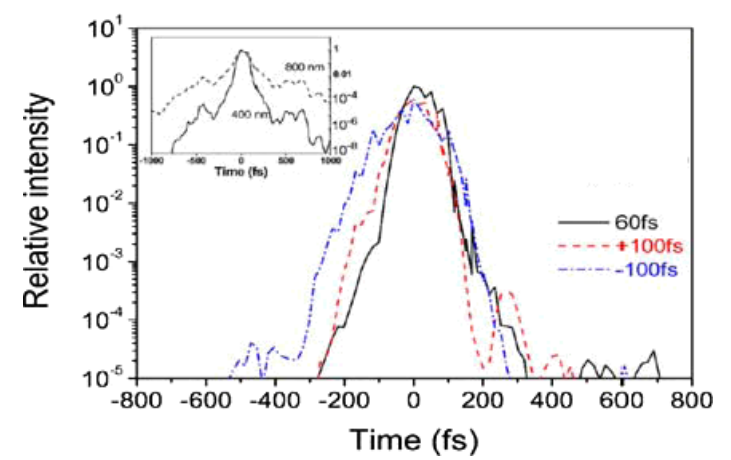

Fig. 11 Temporal pulse shape for the high-contrast 400-nm laser for $60 \mathrm{fs}$ (solid line), positively modified by $100 \mathrm{fs}$ (dashed line), and negatively modified by $100 \mathrm{fs}$ (dashdotted line). The inset shows the temporal pulse profile for the low-contrast 800-nm pulse (dashed line) and the high-contrast 400-nm pulse (solid line).

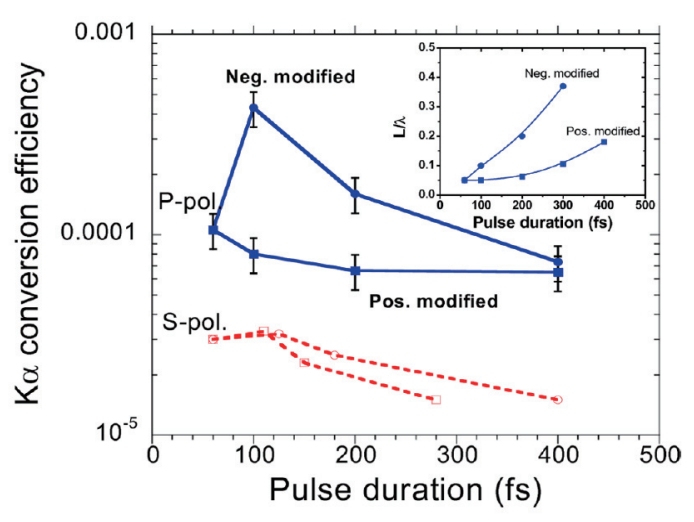

Fig. $12 \mathrm{Cu} \mathrm{K}_{\alpha}$ X-ray conversion efficiency as a function of laser pulse width, negatively modified (solid circle) and positively modified (solid square), for a p-polarized 400-nm laser, and also negatively modified (open circle) and positively modified (open square) for an s-polarized 400-nm laser. The inset shows the corresponding plasma scale length $(L / \lambda)$ before the pulse peak arrives.

sorption mechanism in the experiment. This is further confirmed by PIC simulation and direct experimental observation of the fast electrons generated by $p$ - and $s$-polarized laser pulses. Thus, we find that high-contrast lasers are an effective tool for optimizing x-ray emission by increasing the hot electrons using vacuum heating.

\section{Summary}

Recent progress in fast-electron generation and transport, proton and ion acceleration, and $\mathrm{THz}$ and $\mathrm{x}$-ray emission in intense laser-plasma interactions at the Institute of Physics, Chinese Academy of Sciences is reviewed. With femtosecond laser systems, we have also studied some new topics not inclued in this review, such as the long-distance propagation and filamentation of short laser 
pulses, ultrafast-electron diffraction, laser wakefield particle acceleration, photoionized plasmas, and shock waves related to astrophysics. With the rapid development of high-intensity lasers worldwide, as for the ELI and HYPER projects, more new physics and potential applications will be found.

\section{Acknowledgments}

This work was jointly supported by the Natural Science Foundation of China (Grant No. 60621063, 10734130, 10675164, and 10720101075), the National High-Tech ICF program, and National Basic Research Program of China (973 Program) (Grant No. 2007CB815101).

[1] M. Tabak et al., Phys. Plasmas 1, 1621 (1994).

[2] J. Fuchs et al., Nature Phys. 2, 48 (2006); L. Robson et al., Nature Phys. 3, 58 (2007); B.M. Hegelich et al., Nature 439, 441 (2006); H. Schwoerer et al., Nature 439, 445 (2006); T. Toncian et al., Science 312, 410 (2006).

[3] H. Schwoerer et al., Phys. Rev. Lett. 86, 2317 (2001).

[4] W.L. Kruer et al., Phys. Fluids 28, 430 (1985).

[5] F. Brunel, Phys. Rev. Lett. 59, 52 (1987).

[6] Z.-M. Sheng et al., Phys. Rev. Lett. 88, 055004 (2002).

[7] W.L. Kruer, The Physics of Laser Plasma Interactions (Addison-Wesley publishing company, Redwooddity, California, 1988); S.C. Wilks et al., Phys. Plasmas 2, 274 (1995).

[8] R.B. Stephens et al., Phys. Rev. E 69, 66414 (2004).

[9] T. Feurer et al., Phys. Rev. E 56, 4608 (1997).

[10] P. McKenna et al., Phys. Rev. Lett. 98, 145001 (2007).

[11] Y.T. Li et al., Phys. Rev. Lett. 96, 165003 (2006).

[12] T. Nakamura et al., Phys. Rev. Lett. 93, 265002 (2004).

[13] Y. Sentoku et al., Phys. Plasmas 11, 3083 (2004).

[14] R. Kodama et al., Nature 412, 798 (2001).

[15] X.H. Yuan et al., Phys. Plasmas 15, 013106 (2008).

[16] X.H. Yuan et al., Opt. Express 16, 81 (2008).

[17] R.B. Campbell et al., Phys. Plasmas 10, 4169 (2003).

[18] R. Kodama et al., Nature 423, 1005 (2004).

[19] B.I. Cho et al., Phys. Plasmas 15, 052701 (2008).

[20] Y.T. Li et al., Phys. Rev. E 77, 016406 (2008).

[21] J.R. Davis et al., Phys. Rev. E 56, 7193 (1997).

[22] Y. Sentoku et al., Phys. Rev. Lett. 90, 155001 (2003).

[23] M.S. Wei et al., Phys. Rev. E 70, 056412 (2004) and references in.
[24] M. Honda et al., Phys. Plasmas 7, 1302 (2000).

[25] M. Manclossi et al., Phys. Rev. Lett. 96, 125002 (2006).

[26] M.H. Xu et al., Phys. Plasmas 13, 104507 (2006).

[27] Y.T. Li et al., Phys. Rev. E 72, 066404 (2005).

[28] T. Esirkepov et al., Phys. Rev. Lett. 92, 175003 (2004).

[29] J. Denavit, Phys. Rev. Lett. 69, 3052 (1992).

[30] L.O. Silva et al., Phys. Rev. Lett. 92, 015002 (2004).

[31] M. Chen et al., Phys. Plasmas 14, 053102 (2007).

[32] M. Chen et al., Phys. Plasmas 14, 113106 (2007).

[33] M.-Q. He et al., Phys. Rev. E 76, 035402 (2007).

[34] X.Q. Yan et al., Phys. Rev. Lett. 100, 135003 (2008).

[35] R. Lichters et al., Phys. Plasmas 3, 3425 (1996).

[36] W.P. Leemans et al., IEEE Trans. Plasma Sci. 33, 8 (2005).

[37] M.S. Sherwin, C.A. Schmuttenmaer and P.H. Bucksbaum, Opportunities in $\mathrm{THz}$ Science, Report of a DOE-NSFNIHWorkshop held February 12-14, 2004, Arlington, VA.

[38] G.L. Carr et al., Nature 420, 153 (2002).

[39] W.P. Leemans et al., Phys. Rev. Lett. 91, 074802 (2003).

[40] H. Hamster et al., Phys. Rev. E 49, 671 (1994).

[41] N. Yugami et al., Phys. Rev. Lett. 89, 065003 (2002).

[42] Z.M. Sheng et al., Phys. Rev. Lett. 94, 095003 (2005).

[43] Z.M. Sheng et al., Phys. Plasmas 12, 123103 (2005).

[44] Z.M. Sheng et al., Phys. Rev. E 69, 025401 (2004).

[45] Z.M. Sheng et al., J. Plasma Phys. 72, 795 (2006).

[46] H.C. Wu et al., Phys. Rev. E 75, 016407 (2007).

[47] H.C. Wu et al., Phys. Rev. E 77, 046405 (2008).

[48] D.J. Cook et al., Opt. Lett. 25, 1210 (2000).

[49] K.-Y. Kim et al., Opt. Express 15, 4577 (2007).

[50] V.B. Gildenburg et al., Phys. Rev. Lett. 98, 245002 (2007).

[51] C. D'Amico et al., Phys. Rev. Lett. 98, 235002 (2007).

[52] H.C. Wu, J. Meyer-ter-Vehn and Z.M. Sheng, New J. Phys. 10, 043001 (2008).

[53] W.-M. Wang et al., Opt. Exp. 16, 16999 (2008).

[54] H. Chen et al., Phy. Rev. Lett. 70, 3431 (1993).

[55] A. Krol et al., Med. Phys. 24, 725 (1997).

[56] Ch. Reich et al., Phys. Rev. Lett. 84, 4846 (2000); D. Salzmann et al., Phys. Rev. E 65, 036402 (2002); A. Zhidkov et al., Phys. Rev. E 62, 7232 (2000); M. Schnurer et al., Phys. Rev. E 61, 4394 (2000).

[57] L.M. Chen et al., Phys. Rev. Lett. 100, 045004 (2008).

[58] W.P. Leemans et al., Phys. Rev. Lett. 89, 174802 (2002); U. Andiel et al., Appl. Phys. Lett 80, 198 (2002); T. Guo et al., Rev. Sci. Instrum. 72, 41 (2001). 